\title{
Postoperative Meningitis in Patients with Cervical Cord Tumor: A Case Report
}

\author{
Yoshiharu Kawaguchi, Shoji Seki, Taketoshi Yasuda, \\ Yumiko Nakamura, Masato Nakano, Tomoatsu Kimura \\ Department of Orthopaedic Surgery, Faculty of Medicine, University of Toyama, Toyama, Japan
}

Postoperative meningitis after spinal surgery is a rare complication that can result in a life-threatening condition. Linezolid (LZD) is an oxazolidinone which has been approved in Japan for infections caused by methicillin-resistant Staphylococcus aureus. The authors encountered a case of postoperative meningitis with cerebrospinal fluid leakage (liquorrhoea) that occurred after resection of a cervical cord tumor. The infection was caused by methicillin-resistant Staphylococcus epidermidis (MRSE). Debridement and suture of the dura matter was carried out. LZD was given intravenously. The infection was cured without any sequelae. Based on this result, we concluded that LZD might be considered as one of the first choices for the treatment of postsurgical meningitis caused by MRSE.

Key Words: CSF leakage, Postoperative meningitis, Surgical site infection, Methicillin-resistant Staphylococcus epidermidis, Linezolid

\section{Introduction}

Postoperative spinal infection is an unfavorable complication, and superficial and deep wound infections are included in this category. In a survey of postoperative spinal infections that included more than 2000 cases of spinal operation, the overall infection rate was reported to be $1.9 \%$ [1]. Postoperative meningitis after spinal surgery is a rare complication that can result in a life-threatening condition. Several therapeutic methods have been employed for treating postoperative meningitis. Linezolid (LZD) is an oxazolidinone, which is a new class of antibiotics [2]. It has been approved in Japan for infections caused by methicillinresistant Staphylococcus aureus. LZD has a higher rate of penetration rate into the cerebrospinal fluid (CSF) than vancomycin (VCM) [3]. Previous reports have described the efficacy of LZD for central nervous system infection, including postoperative meningitis after intracranial surgery [3-8]. We present here a case of postoperative meningitis with CSF leakage (liquorrhoea) that occurred after resection of a cervical cord tumor. The infection was caused by methicillin-resistant Staphylococcus epidermidis (MRSE). Debridement and suture of the dura matter was carried out. Subarachnoid drainage from the lumbar spine was also done and LZD was given intravenously. The infection was cured without any sequelae. These treatments were considered to be effective in this patient.

\section{Case Report}

A 39-year-old woman complained of neck pain and left scapular pain in January 2006. The symptoms gradually increased and she was eventually admitted to our hospital.

Received Feb 9, 2010; 1st Revised Feb 27, 2010; Accepted Mar 2, 2010

Corresponding author: Yoshiharu Kawaguchi, MD

Department of Orthopaedic Surgery, Faculty of Medicine, University of Toyama,

2630 Sugitani, Toyama 930-0194, Japan

Tel: +81-76-434-7353, Fax: +81-76-434-5035, E-mail: zenji@med.u-toyama.ac.jp 


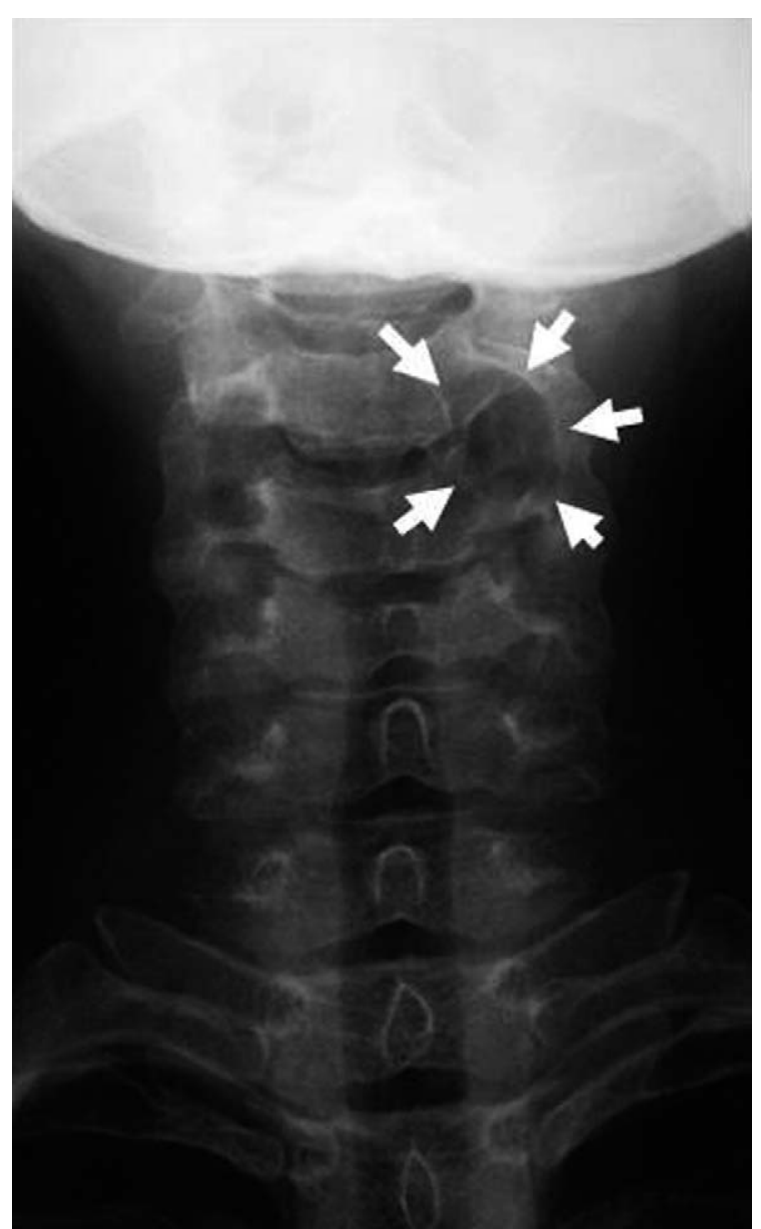

Fig. 1. Anteroposterior radiograph of the cervical spine. Enlargement of the left C3-4 foramen was observed (arrows).
Physical examination revealed deep tendon hyper-reflexia of the upper and lower extremities. Motor palsy was not evident and no sensory disturbance was found in the upper or lower extremities or in the trunk. The plain lateral radiography did not show any abnormality. The antero-posterior radiography showed enlargement of the left C3-4 foramen (Fig. 1). Cervical magnetic resonance imaging demonstrated a dumbbell type tumor compressing the spinal cord at the C3-4 levels (Fig. 2). The tumor was located on the left side of the spinal cord. The tumor showed low intensity on the T1-weighted image and iso-high or slightly high intensity on the T2-weighted image. Gadolinium enhancement was noted in the tumor (Fig. 2).

The patient underwent an operation in April 2006. Cervical laminectomy at C3-4 was carried out and total resection of the cervical tumor was performed. The tumor was encapsulated (Fig. 3) and it involved the posterior ramus of the C4 spinal nerve, and so the nerve was resected. The dura mater was opened to identify the presence of any intradural tumor invasion, but no intradural invasion was observed. Thereafter, the dura matter was tightly sutured. The roundshaped dark red tumor mass was covered by a thin membrane. The size of the tumor was $2.6 \times 2.4 \times 1.3 \mathrm{~cm}$. The histological examination revealed a schwanoma with both Antoni type A and type B areas. No malignant characteristics were found for the pathology.

The surgical drain was removed 2 days after surgery. The patient had a slight fever. On the 9 th postoperative day, she
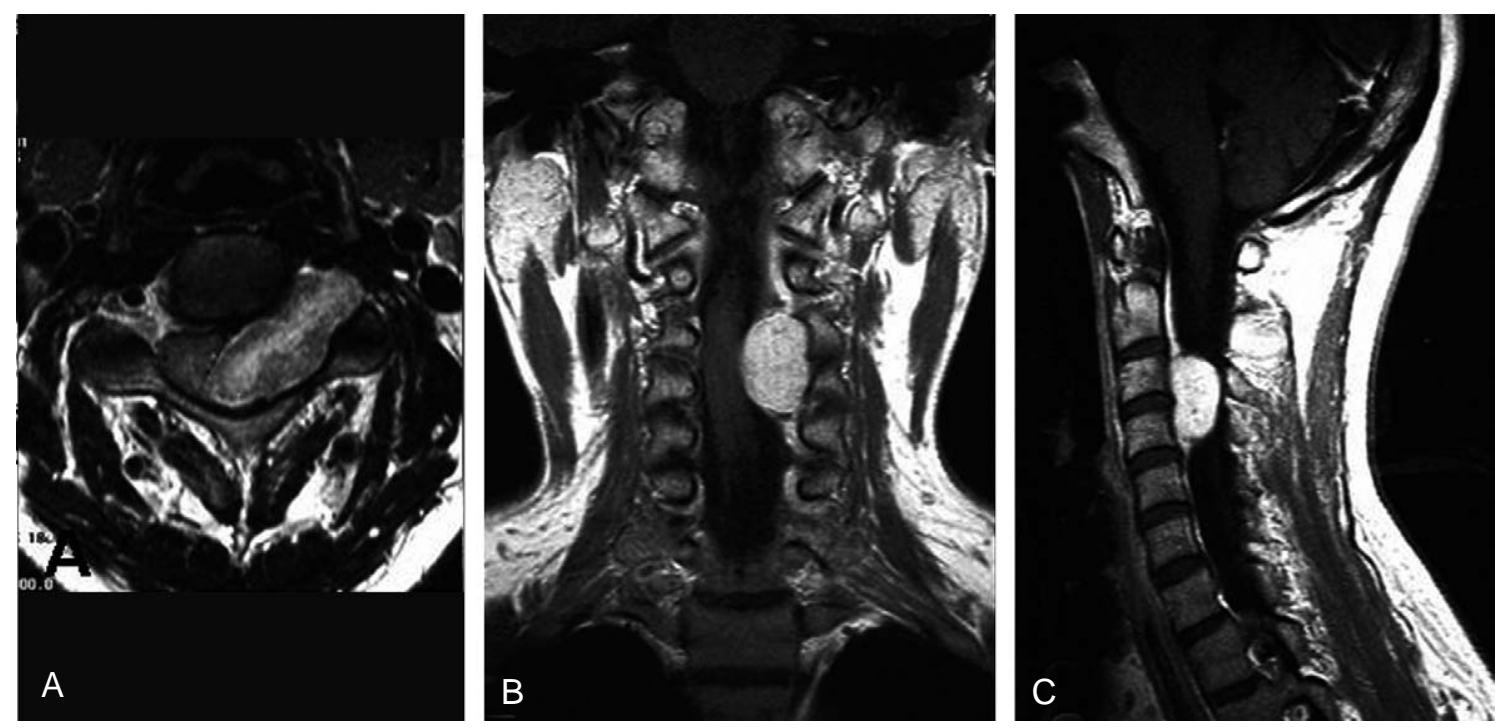

Fig. 2. Preoperative magnetic resonance imaging of the cervical spine. The axial T2 image revealed a dumbbell type tumor compressing the spinal cord at the C3-4 levels (A). The gadolinium-enhanced frontal (B) and sagittal images (C) indicated that the tumor was markedly enhanced. 
displayed a high grade fever $\left(>39.0^{\circ} \mathrm{C}\right)$ and vomiting. The Kernig sign was observed. She had fluctuation near the surgical wound, and so a spinal cord puncture was carried out; $25 \mathrm{ml}$ of turbid CSF was obtained near the surgical wound. Culture of the CSF revealed MRSE. We diagnosed that she was suffering with postoperative meningitis. The patient also had liquorrhoea. Debridement was performed and the laceration of dura matter was sutured on the 18th day after the first surgery. The size of laceration was $1 \mathrm{~mm}$ in diameter at the site where the posterior ramus of the $\mathrm{C} 4$ spinal nerve was resected (Fig. 4). She had drainage from the lumbar spine for 1 week after the second surgery. As for chemotherapy, she received intravenous administration of $600 \mathrm{mg}$ of LZD twice a day from the 11th day to the 20th day after the first surgery, and the same dose of LZD was given from the 5th day to the 12th day after the second surgery. These treatment methods, including the second surgery, spinal drainage and chemotherapy, were very effective. The patient did not have fever thereafter and the infection healed. She was discharged on the 18th day after second surgery (Fig. 5). Thereafter, she has had an excellent postoperative course and no recurrence of infection or tumor was observed for the following 3 years.

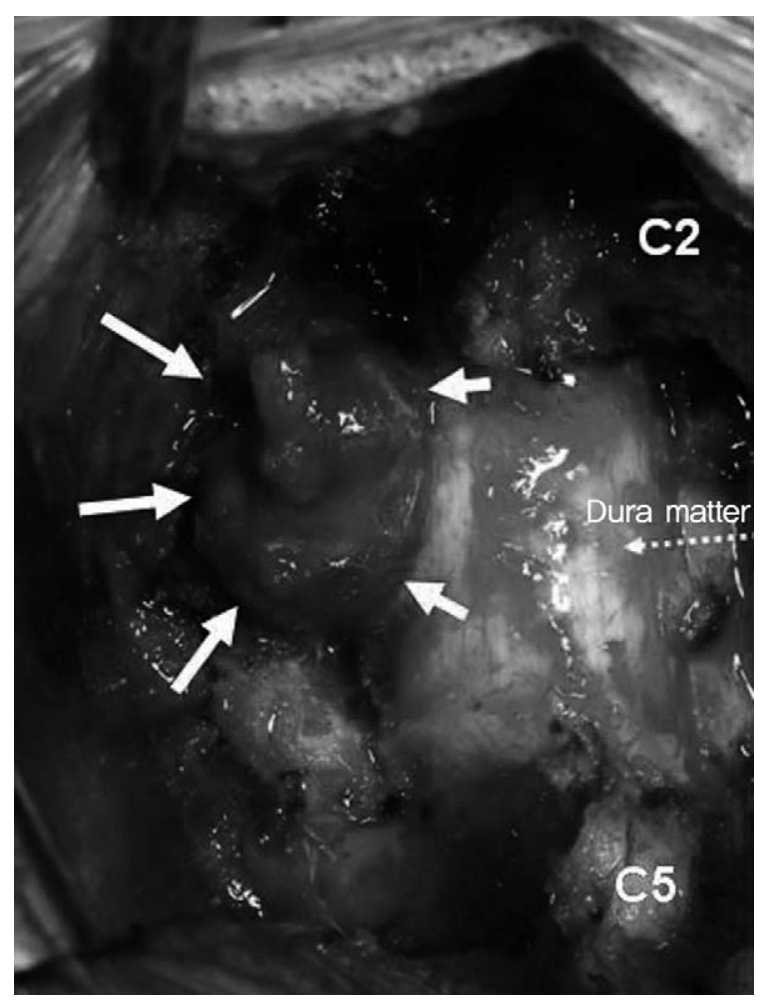

Fig. 3. Intraoperative findings of the tumor. The tumor was encapsulated by a thin membrane (arrows).

\section{Discussion}

We present here an unusual case of postoperative meningitis that was caused by MRSE following cervical cord tumor resection. Surgical infection is rarely encountered after spine surgery, yet therapeutic problems occur when it is encountered. Weinstein et al. [1] described that among 2391 operative procedures of the spine, 46 cases of wound infection were identified, yielding an overall infection rate of $1.9 \%$. A multicenter study in the USA revealed that $11.9 \%$ of the patients with neuromuscular scoliosis had deep wound infection after surgery [9]. Although the incidence of postoperative meningitis after spine surgery is unknown, this infectious condition is thought to be very rare. Regarding the mechanism of postoperative meningitis in the present case, it is speculated that the infection occurred at the surgical site and it spread into the intradural space. We opened the dura matter because we anticipated the tumor had invaded to the intradural space. The opened region was then firmly sutured. Although there was no neural deficit after resection of the $\mathrm{C} 4$ nerve root, the liqu-

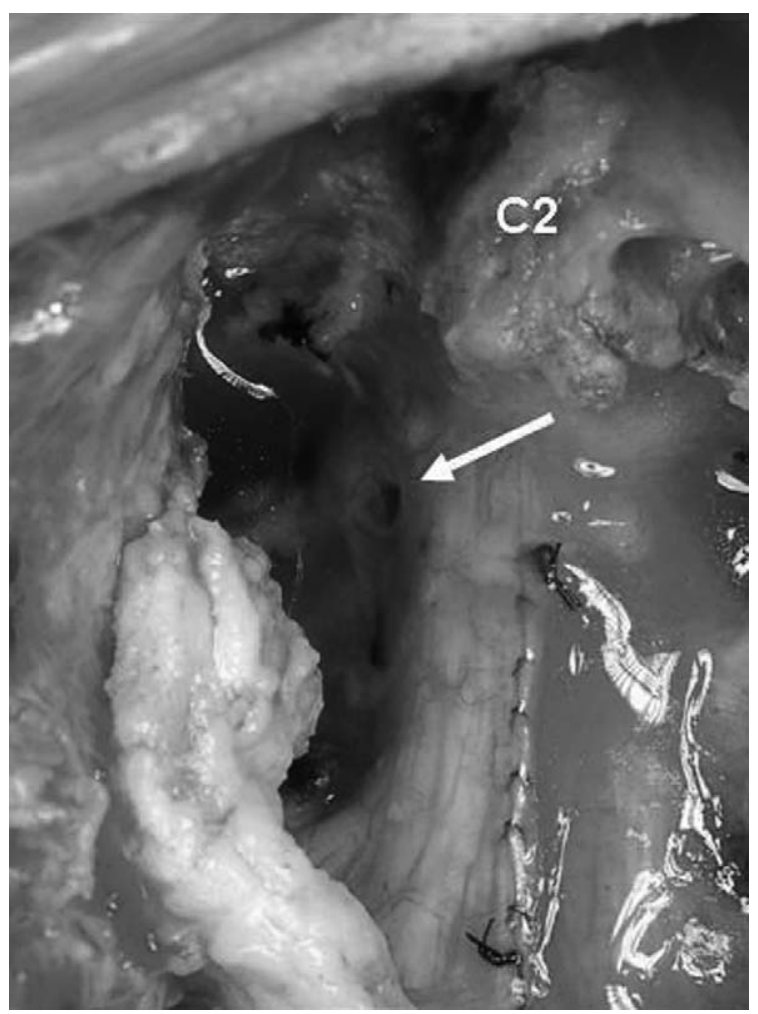

Fig. 4. Intraoperative findings at the second surgery. The defect of the dura matter was found and liquorrhoea through the defect was observed (arrow). The defect was tightly sutured. 
Human immunoglobulin $2.5 \mathrm{~g} /$ day for 3 days

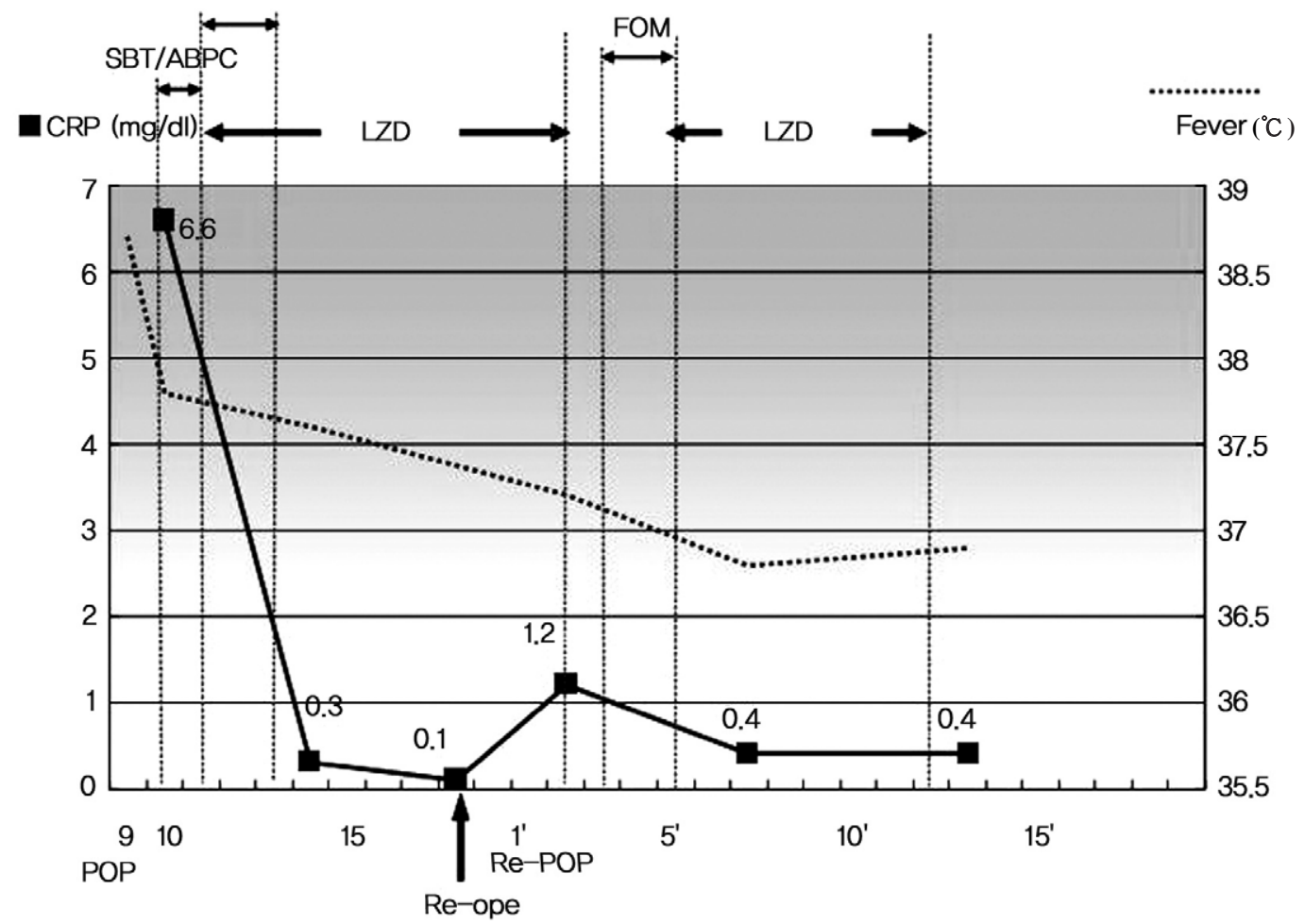

Fig. 5. The pre- and postoperative clinical course at the time of the second surgery. The patient's condition was favorable after the second surgery. POP: Postoperation, CRP: C-reactive protein, SBT/ABPC: Sulbactam/ampicillin, FOM: Fosphomycin, LZD: Linezolid.

orrhoea through the dural defect from the resection of the C4 spinal nerve and the dead space from the removal of the tumor might have been the background of the infection. Several papers have pointed out that liquorrhoea might cause the formation of a dural cutaneous fistula and this increases the risk of postoperative meningitis [10-12]. Those reports emphasized that repair of the injured dura matter and lumbar subarachnoid drainage should be carried out as a means of preventing meningitis or pseuromeningocele.

We used LZD as chemotherapy against the MRSE meningitis in the present case. It proved to be very effective for the treatment, based on the patient's favorable clinical and laboratory course after the occurrence of infection. LZD is an oxazolidinone, which is a new class of antibiotics. It has shown consistent inhibitory activity against multiresistant Staphylococci, Enterococci and Pneomococci [13]. LZD selectively binds to the $50 \mathrm{~S}$ ribosomal subunit near the interface with the $30 \mathrm{~S}$ subunit, which results in a distortion that inhibits the formation of the initiation complex [2]. Intravenous administration of VCM or teicoplanin (TEIC) is generally considered as first line treatment for methi- cillin-resistant gram positive cocci. However, intravenous administration of VCM and TEIC results in a low concentration of drug in the CSF. The ratios between the concentrations of antibiotic in the CSF and plasma have been reported as $18-40 \%$ for VCM [14] and less than $10 \%$ for TEIC [15]. On the other hand, the ratio is extremely high for the intravenous administration of LZD. It was reported to be $160 \%$ on average, and it ranged from $120 \%$ to $230 \%$ [3]. Thus, intravenous administration of LZD has an advantage for the treatment of meningitis induced by methicillinresistant gram positive cocci. In fact, there have been two case reports that discussed the beneficial effect of LZD against the postneurosurgical meningitis caused by MRSE after cranial surgery [5,6] and where intravenous VCM failed to eradicate the infection. LZD might be considered as one of the first choices for the treatment of postsurgical meningitis caused by MRSE.

\section{REFERENCES}

1. Weinstein MA, McCabe JP, Cammisa FP Jr. Postoperative spinal wound infection: a review of 2,391 consecutive 
index procedures. J Spinal Disord 2000;13:422-6.

2. Corti G, Cinelli R, Paradisi F. Clinical and microbiologic efficacy and safety profile of linezolid, a new oxazolidinone antibiotic. Int J Antimicrob Agents 2000;16:527-30.

3. Villani P, Regazzi MB, Marubbi F, et al. Cerebrospinal fluid linezolid concentrations in postneurosurgical central nervous system infections. Antimicrob Agents Chemother 2002;46:936-7.

4. Viale P, Pagani L, Cristini F, et al. Linezolid for the treatment of central nervous system infections in neurosurgical patients. Scand J Infect Dis 2002;34:456-9.

5. Nagashima G, Okamoto N, Okuda M, et al. Effect of linezolid against postneurosurgical meningitis caused by methicillin-resistant Staphylococcus epidermidis: case report. J Infect Chemother 2008;14:147-50.

6. Krueger WA, Kottler B, Will BE, Heininger A, Guggenberger H, Unertl KE. Treatment of meningitis due to methicillin-resistant Staphylococcus epidermidis with linezolid. J Clin Microbiol 2004;42:929-32.

7. Shaikh ZH, Peloquin CA, Ericsson CD. Successful treatment of vancomycin-resistant Enterococcus faecium meningitis with linezolid: case report and literature review. Scand J Infect Dis 2001;33:375-9.

8. Ntziora F, Falagas ME. Linezolid for the treatment of patients with central nervous system infection. Ann Pharmacother 2007;41:296-308.

9. Sponseller PD, LaPorte DM, Hungerford MW, Eck K,
Bridwell KH, Lenke LG. Deep wound infections after neuromuscular scoliosis surgery: a multicenter study of risk factors and treatment outcomes. Spine (Phila Pa 1976) 2000;25:2461-6.

10. Eismont FJ, Wiesel SW, Rothman RH. Treatment of dural tears associated with spinal surgery. J Bone Joint Surg Am 1981;63:1132-6.

11. Kitchel SH, Eismont FJ, Green BA. Closed subarachnoid drainage for management of cerebrospinal fluid leakage after an operation on the spine. J Bone Joint Surg Am 1989;71:984-7.

12. Shapiro SA, Scully T. Closed continuous drainage of cerebrospinal fluid via a lumbar subarachnoid catheter for treatment or prevention of cranial/spinal cerebrospinal fluid fistula. Neurosurgery 1992;30:241-5.

13. Livermore DM. Quinupristin/dalfopristin and linezolid: where, when, which and whether to use? J Antimicrob Chemother 2000;46:347-50.

14. Albanèse J, Léone M, Bruguerolle B, Ayem ML, Lacarelle B, Martin C. Cerebrospinal fluid penetration and pharmacokinetics of vancomycin administered by continuous infusion to mechanically ventilated patients in an intensive care unit. Antimicrob Agents Chemother 2000;44:1356-8.

15. Stahl JP, Croize J, Wolff M, et al. Poor penetration of teicoplanin into cerebrospinal fluid in patients with bacterial meningitis. J Antimicrob Chemother 1987;20:141-2. 